\title{
Nanophotonic Platforms for Enhanced Chiral Sensing
}

\author{
E. Mohammadi, ${ }^{\dagger}{ }^{\ddagger}$ K. L. Tsakmakidis, ${ }^{\dagger}$ A. N. Askarpour, ${ }^{\dagger}$ P. Dehkhoda, ${ }^{\dagger}$ A. Tavakoli, ${ }^{\ddagger}$ and H. Altug ${ }^{*}{ }^{\dagger}$ \\ †Bioengineering Department, École Polytechnique Fédérale de Lausanne (EPFL), 1015 Lausanne, Switzerland \\ ${ }^{\ddagger}$ Department of Electrical Engineering, Amirkabir University of Technology, Tehran, Iran
}

\section{Supporting Information}

ABSTRACT: Chirality plays an essential role in life, providing unique functionalities to a wide range of biomolecules, chemicals, and drugs, which makes chiral sensing and analysis critically important. The wider application of chiral sensing continues to be constrained by the involved chiral signals being inherently weak. To remedy this, plasmonic and dielectric nanostructures have recently been shown to offer a viable route for enhancing weak circular dichroism (CD) effects, but most relevant studies have thus far been ad hoc, not guided by a rigorous analytical methodology. Here, we report the first
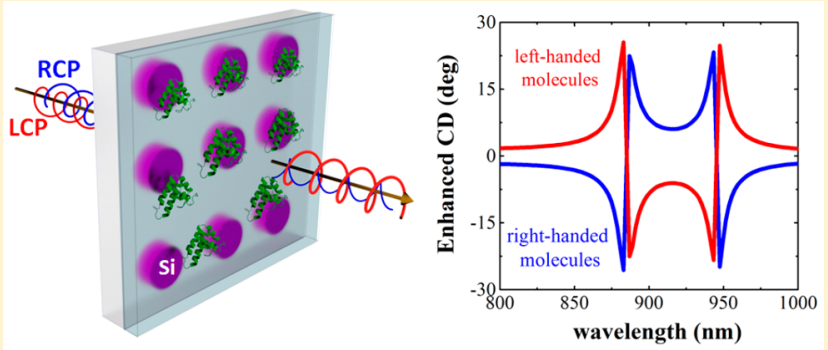
analytical treatment of $\mathrm{CD}$ enhancement and extraction from a chiral biolayer placed on top of a nanostructured substrate. We derive closed-form expressions of the $\mathrm{CD}$ and its functional dependence on the background-chiroptical response, substrate thickness and chirality, as well as on the optical chirality and intensity enhancement provided by the structure. Our results provide key insights into the trade-offs that are to be accommodated in the design and conception of optimal nanophotonic structures for enhancing CD effects for chiral molecule detection. Based on our analysis, we also introduce a practical, dielectric platform for chiral sensing featuring large $\mathrm{CD}$ enhancements while exhibiting vanishing chiroptical background noise.

KEYWORDS: chirality, chiral sensing, circular dichroism, nanophotonics

$\mathrm{C}$ hirality is a ubiquitous phenomenon in nature and plays an essential role in life. ${ }^{1}$ Today, we know that biological functionality of chiral biomolecules (e.g., proteins, amino acids, and carbohydrates), as well as synthesized chiral molecules in drugs, is strongly influenced by their chirality. ${ }^{2,3}$ As a result, discrimination of enantiomers is indeed critically important in biology and the pharmaceutical industry in terms of safety and production costs. ${ }^{4,5}$ Enantiomers are distinguishable in the way they interact with chiral fields. Traditionally, circular dichroism (CD) spectroscopy has been used for this discrimination, which is essentially the differential absorption for circularly polarized light (CPL). ${ }^{6,7}$ However, the main challenge of CD spectroscopy still remains the weak chiroptical signals, due to the weak chirality of matter ${ }^{8}$ interacting with plane wave lightfields.

With the advent of plasmonics and metamaterials, ${ }^{9,10}$ finding new ways and structures to enhance these weak chirality effects has been an area of extensive research. ${ }^{11-14} \mathrm{~A}$ wide range of structures, including three-dimensional nanohelix arrays, ${ }^{15-17}$ twisted crosses ${ }^{18}$ and twisted nanorods, ${ }^{19}$ chiral oligomers ${ }^{20}$ and chiral assemblies of nanoparticles, ${ }^{21,22}$ single $^{23}$ or multilayer gammadion arrays, ${ }^{24}$ and even more complicated structures, such as intertwined 3D helices, ${ }^{25}$ have been investigated for enhancing chiroptical effects. Such nanophotonic structures can be realized by bulk metamaterials ${ }^{15-17}$ or thin planar metasurfaces, ${ }^{23}$ chiral $^{15-25}$ or achiral ${ }^{26,27}$ inclusions, and plasmonic $^{15-25}$ or dielectric ${ }^{28}$ media. When a chiral sample is placed in the near-field of such nanostructures, the CD signal can be enhanced according to the enhancement in the field intensity and optical chirality ${ }^{29,30}$ provided by the nano- photonic platform. ${ }^{31,32}$ Among these two enhancement features, the formation of highly chiral near-fields close to the nanophotonic structure, plays an essential role in enhancing the CD signal. However, it is important to note that all of these platforms have their own intrinsic chiroptical response, which appears as an additive term in the final CD signal and acts as a background noise for the chiral response of biolayers.

So far, the extensive studies of chiral nanophotonic platforms have been mainly pursued numerically or experimentally, ascertaining the performance of the final device from the intrinsic (without the biolayer) chiroptical response of the substrate (such as, its intrinsic CD and provided optical chirality). ${ }^{33}$ Yet, the connection of these inherent characteristics of chiral substrate to the CD signal in the presence of a biolayer (necessary for chiral sensing) still remains elusive. Furthermore, several simplified systems have been studied analytically. ${ }^{22,34}$ It is the objective of this paper to report the first analytic study of the problem of substrate CD spectroscopy for an arbitrary nanophotonic substrate (either, chiral or achiral, plasmonic or dielectric) and clarify the interplay between key affecting parameters, such as the thickness and chirality of the substrate, as well as the near-field optical chirality enhancement. We formulate a simple analytical description of the phenomenon elucidating the involved trade-offs, such as between the choice of bulk metamaterials or thin planar metasurfaces, chiral or

Received: February 27, 2018

Published: May 22, 2018 
(a)

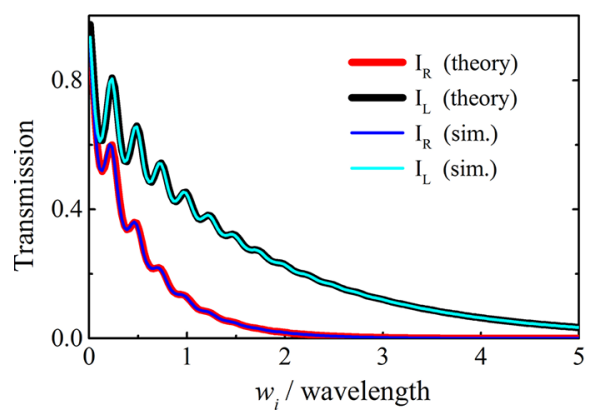

(b)

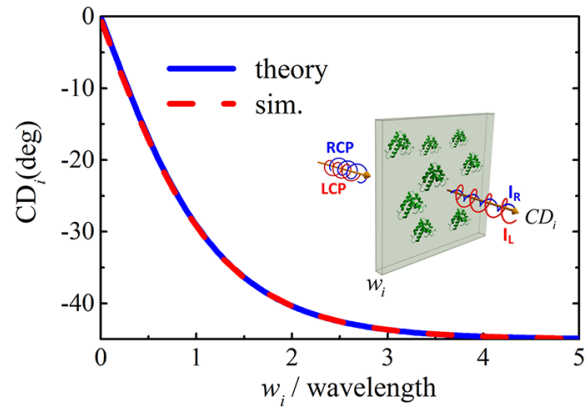

Figure 1. Conventional CD spectroscopy. (a) Transmission intensities of RCP $\left(I_{\mathrm{R}}\right)$ and LCP $\left(I_{\mathrm{L}}\right)$ waves for a chiral biolayer of refractive index $n_{\mathrm{i}}=$ $2-0.1 j$ and Pasture parameter of $\kappa_{\mathrm{i}}=0.1-0.05 j$, as a function of the biolayer's thickness $\left(w_{\mathrm{i}}\right)$. (b) $\mathrm{CD}_{\mathrm{i}}$ signal corresponding to the transmission spectra in (a), calculated from eq 1 . The inset shows the chiral sample made of chiral biomolecules and illuminated by RCP (blue) and LCP (red) lightfields of equal intensities.

(a)

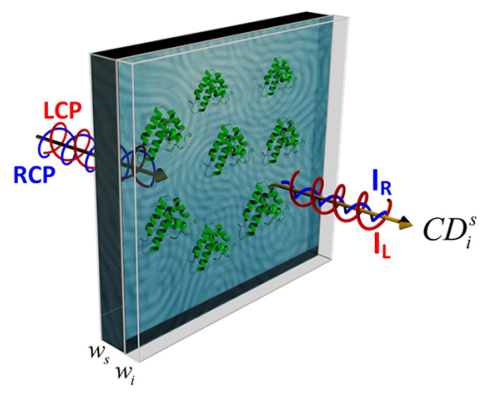

(b)

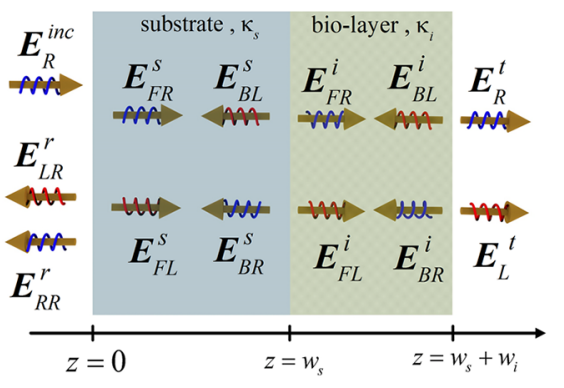

(c)

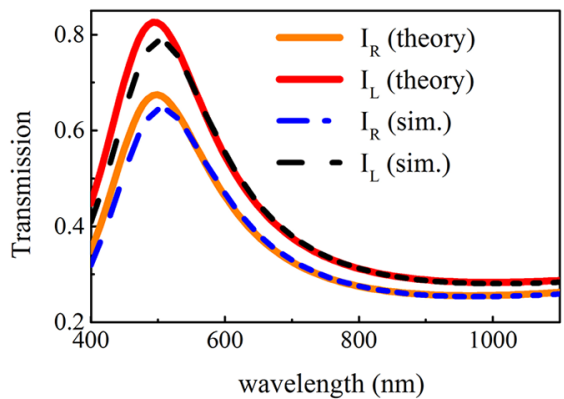

(d)

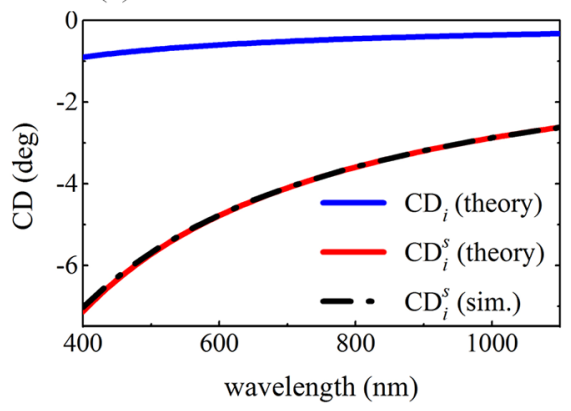

(e)

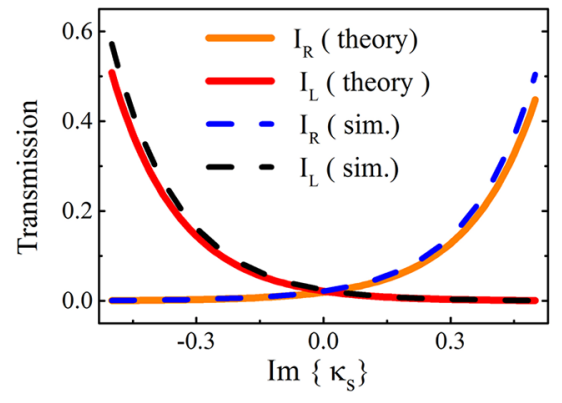

(f)

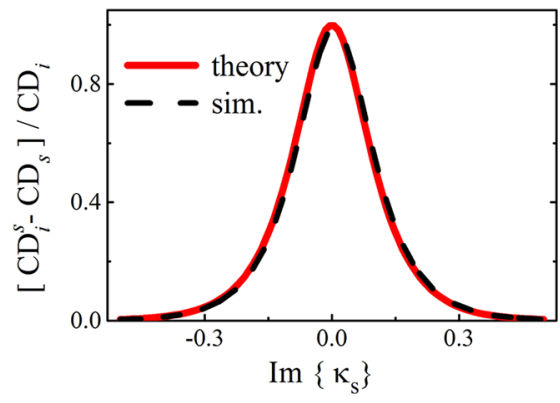

Figure 2. (a) A chiral biolayer of thickness $w_{i}$ placed on top of a simple chiral substrate of thickness $w_{s}$, with the whole system being excited by RCP (blue) and LCP (red) light. (b) The configuration in (a), modeled as two homogeneous juxtaposed chiral layers of thicknesses and Pasture parameters of $w_{i} \kappa_{i}$ (for biolayer) and $w_{s}, \kappa_{\mathrm{s}}$ (for substrate), placed in free space and illuminated under RCP light excitation. With their corresponding nomenclature, the incidence (inc), reflected $(\mathrm{r}$ ), internal ( $\mathrm{s}$ for substrate and $\mathrm{i}$ for biolayer), and transmitted ( $\mathrm{t}$ ) electric field waves are shown schematically. R (L) stands for right (left) circular polarization and F (B) stands for forward (backward) propagating light. (c) Transmission intensities and (d) $C D_{\mathrm{i}}^{s}$ for a biolayer of thickness $10 \mathrm{~nm}$ placed on top of a substrate of thickness $70 \mathrm{~nm}$, where the refractive indices and Pasture parameters are assumed to be $n_{\mathrm{i}}=2-.1 j, \kappa_{i}=0.2-0.05 j$ (biolayer), and $n_{\mathrm{s}}=3.5-.1 j, \kappa_{\mathrm{s}}=0.5-0.05 j$ (substrate). The blue curve indicates the CD signal of the individual biolayer. (e) Transmission intensities for biolayer of thickness $\lambda / 10$ and $\kappa_{\mathrm{i}}=0.2-0.05 j$ placed on top of a substrate of thickness $\lambda / 2$, as a function of $\operatorname{Im}\left\{\kappa_{\mathrm{s}}\right\}$. (f) Normalized desired CD signal of the chiral biofilm as a function of $\operatorname{Im}\left\{\kappa_{\mathrm{s}}\right\}$.

achiral structures, and between choosing plasmonic or dielectric media.

The paper is organized as follows: In section I, we first analyze an isolated, single chiral biolayer. Then, we proceed by retrieving the $\mathrm{CD}$ signal from the same biolayer placed on top of a simple chiral substrate that does not enhance the field intensity or chirality in its near-field zone. On the basis of derived analytical expressions, we report a newly identified consequence of substrate chiral absorption (besides background CD noise), which appears in the form of an attenuation factor acting on the CD signal of the biolayer. In section II, we generalize the analytical results to nanophotonic substrates by introducing intensity and optical chirality enhancement factors. Based on our analytical results, we propose practical ways to remove the background chiroptical noise of the substrate, as well as eliminating the dependency of the output $\mathrm{CD}$ on the permittivity of the biolayer. Finally, we introduce a practical dielectric platform for chiral sensing offering unique advantages including an enhanced CD signal which is only associated with the molecular handedness, while featuring vanishing chiroptical background response. We will show that the CD enhancement originates from highly chiral near-fields provided by the electric and magnetic dipolar resonances of the dielectric structures. 
I. CD Signal of a Biolayer Placed on Top of a Simple Chiral Substrate. In substrate CD spectroscopy, the biolayer is placed on top of a substrate, and the whole system is illuminated by right-circularly polarized (RCP) and leftcircularly polarized (LCP) light sequentially. The CD signal is defined as the differential absorption (transmission) of the light for these two polarization states. If $I_{\mathrm{R}}$ and $I_{\mathrm{L}}$ are the transmitted power intensities for RCP and LCP excitations at the output side of the biolayer, respectively, then the CD signal is defined as ${ }^{32}$

$$
\mathrm{CD}=\tan ^{-1}\left(\frac{I_{\mathrm{R}}-I_{\mathrm{L}}}{I_{\mathrm{R}}+I_{\mathrm{L}}}\right)
$$

First, we consider a conventional CD spectroscopy case, where the $\mathrm{CD}$ signal is retrieved from a chiral biolayer without any substrate. The chiral sample is modeled as a homogeneous slab of thickness $w_{\mathrm{i}}$, placed in free space (inset of Figure 1b) and described by the constitutive equations

$$
\begin{aligned}
& \boldsymbol{D}=\varepsilon_{\mathrm{i}} \boldsymbol{E}-j \kappa_{\mathrm{i}} \sqrt{\varepsilon_{0} \mu_{0}} \boldsymbol{H} \\
& \boldsymbol{B}=\mu_{\mathrm{i}} \boldsymbol{H}+j \kappa_{\mathrm{i}} \sqrt{\varepsilon_{0} \mu_{0}} \boldsymbol{E}
\end{aligned}
$$

where $\varepsilon_{\mathrm{i}}$ and $\mu_{\mathrm{i}}$ are the permittivity and permeability of the chiral sample, respectively, and $\kappa_{\mathrm{i}}$ is the so-called Pasture parameter, $^{35}$ which denotes the material chirality and has different sign for right- and left-handed chiral media. In order to calculate the $\mathrm{CD}$ signal analytically, we find the transmission amplitudes for RCP and LCP excitations separately at normal incidence by solving a boundary value problem expressed in an $8 \times 8$ matrix. The detailed description can be found in the Supporting Information (SI). By inserting the calculated transmitted intensities in eq 1 , we arrive at the following analytical expression for the $\mathrm{CD}$ response of the biolayer

$$
\mathrm{CD}_{\mathrm{i}}=-\tan ^{-1}\left[\tanh \left(2 k_{0} w_{\mathrm{i}} \operatorname{Im}\left\{\kappa_{\mathrm{i}}\right\}\right)\right]
$$

Figure 1a shows the transmission spectra of a typical chiral biolayer as a function of its thickness. The optical parameters of the biolayer are given in the caption of Figure 1. To validate the analytical expressions throughout the paper, we performed fullwave simulations with COMSOL Multiphysics which uses finite element method (FEM). It should be noted that the default built-in fully vectorial wave equations in COMSOL should be modified according to the constitutive relations for a chiral media. ${ }^{36}$ In Figure $1 \mathrm{~b}$ we present the $\mathrm{CD}_{\mathrm{i}}$ signal corresponding to the transmission spectra of Figure la. The $\mathrm{CD}$ signal increases with the thickness of the biofilm, owing to the respective increase of the effective length of the light-matter interaction. The curve reaches a plateau for larger biofilm thicknesses, because chiral loss ${ }^{37}$ attenuates (the transmission of) both CPL polarizations to zero (cf. Figure 1a). Considering that the chiral layer is normally very thin (i.e., $k_{0} w_{\mathrm{i}} \ll 1$ ), we may approximate eq 3 , by keeping only the first two leading terms, as

$$
\mathrm{CD}_{\mathrm{i}}=-2 k_{0} w_{\mathrm{i}} \operatorname{Im}\left\{\kappa_{\mathrm{i}}\right\}
$$

Next, we study the effect of juxtaposing a chiral substrate to the chiral biofilm. As it will become apparent in section II, this intermediate step is useful for analytically identifying the parameters affecting substrate-enhanced CD spectroscopy. Figure $2 \mathrm{a}$ shows a chiral sample, now placed on top of a simple chiral substrate. To solve this problem analytically, we first assume a simple homogeneous chiral matter for the substrate. Here, a "simple" chiral substrate refers to one that does not enhance the intensity or chirality of the light in its near-field zone, as opposed to plasmonic or metamaterial chiral substrates (see section II). In our analysis (cf. Figure $2 b$ ), the configuration of Figure 2a is modeled as two homogeneous juxtaposed chiral slabs of thicknesses $w_{\mathrm{i}}$ (for the biolayer) and $w_{\mathrm{s}}$ (for the substrate), placed in free space. Compared to the biofilm alone (see Figure 1), with the substrate we need to solve a $12 \times 12$ system of boundary-value equations (see SI). Provided that $k_{0} w_{i} \ll 1$, the following compact expression for the output $\mathrm{CD}$ signal is obtained

$$
\mathrm{CD}_{\mathrm{i}}^{\mathrm{s}}=\mathrm{CD}_{\mathrm{s}}+\mathrm{CD}_{\mathrm{i}} \operatorname{sech}\left(4 k_{0} w_{\mathrm{s}} \operatorname{Im}\left\{\kappa_{\mathrm{s}}\right\}\right)
$$

where $\kappa_{\mathrm{s}}$ is the Pasture parameter of the substrate.

Equation 5 provides the total output $\mathrm{CD}_{i}^{s}$ of the structure in terms of individual $\mathrm{CD}$ responses of the substrate $\left(\mathrm{CD}_{\mathrm{s}}\right)$ and the biolayer $\left(C D_{i}\right)$. Here, $C D_{i}$ is given by eq 3 , and $C D_{s}$ pertains to the differential absorption characterizing the substrate without any biolayer, which can be obtained by inserting the corresponding parameters of the substrate $\left(\kappa_{\mathrm{s}}, w_{\mathrm{s}}\right)$ into eq 3. It is, now, evident from eq 5 that placing the biolayer on top of a chiral substrate has two main drawbacks. First, the $\mathrm{CD}_{\mathrm{s}}$ signal of the substrate appears as an additive term in the total output of $\mathrm{CD}_{i}^{s}$ and does not contain any information about the handedness of the biolayer. Thus, it can be considered as an unwanted signal overwhelming the desired sensed signal, which is $\mathrm{CD}_{i}^{s}-\mathrm{CD}_{s}$. The second drawback can be inferred from the second term of eq 5. The multiplication of the $\mathrm{CD}_{\mathrm{i}}$ signal with the sech term, reveals a hitherto-unidentified consequence of chiral loss in substrate CD spectroscopy: as the sech function decreases with increasing argument, the desired sensed signal decreases with the increasing $k_{0} w_{\mathrm{s}} \operatorname{Im}\left\{\kappa_{\mathrm{s}}\right\}$ term. More specifically, the sech term appears because the RCP and LCP excitations pass through the substrate and arrive in the entrance side of the chiral sample with different attenuation factors exerted on them by the substrate. Thus, increasing the optical thickness $\left(k_{0} w_{\mathrm{s}}\right)$ or the imaginary part of $\kappa_{\mathrm{s}}$ not only increases the background noise $\left(\mathrm{CD}_{\mathrm{s}}\right)$, but also decreases the desired sensed signal from the biolayer.

A discussion about realistic solutions to this problem is presented in section II, where specifically we show how nanophotonic platforms can remove the background noise (first drawback), as well as compensate for the attenuation factor of the sech function (second drawback). Figure $2 \mathrm{c}$ and $\mathrm{d}$ show, respectively, the transmission spectra and $\mathrm{CD}_{\mathrm{i}}^{\mathrm{s}}$ signal for a biolayer of thickness $10 \mathrm{~nm}$ placed on top of a substrate of thickness $70 \mathrm{~nm}$. It can be seen that the total CD (black/red) has been enhanced compared to the single biolayer case (blue), but this enhancement originates from $\mathrm{CD}_{s}$ and cannot be attributed to the handedness of the biolayer. In order to further clarify the effect of chiral loss (due to $4 k_{0} w_{s} \operatorname{Im}\left\{\kappa_{s}\right\}$ term) from the substrate on the desired CD signal $\left(\mathrm{CD}_{\mathrm{i}}^{s}-\mathrm{CD}_{\mathrm{s}}\right)$, in Figures $2 \mathrm{e}, \mathrm{f}$ we have studied the problem as a function of the imaginary part of the substrate Pasture parameter. In Figure 2e, the transmission intensities are obtained for a biolayer of thickness $\lambda / 10$ placed on top of a substrate of thickness $\lambda / 2$. The imaginary part of $\kappa_{\mathrm{s}}$ changes between -0.5 and +0.5 . Figure $2 \mathrm{f}$ shows the normalized desired CD signal corresponding to the transmission intensities in Figure 2e, which is in perfect agreement with the sech function, as previously indicated analytically in eq 5 . It can be seen that the simulations and the theoretical results are in excellent agreement. From Figure $2 \mathrm{f}$ 
and eq 5, we can readily infer that substrate Pasture parameter with a nonzero imaginary part (i.e., chiral loss) reduces the desired CD signal.

II. CD Signal of a Biolayer Placed on Top of a Nanophotonic Chiral Substrate. Next, we extend our analysis to nanophotonic (nanostructured) substrates, which have been studied numerically and experimentally in literature. ${ }^{17-27}$ In order for our analytical formulas in this section to remain valid for chiral or achiral, plasmonic or dielectric substrates we consider the most general case of a plasmonic chiral nanophotonic substrate with a chiral biofilm on top.

One of the main difficulties in the analytical treatment of this chiral system is the intricate interaction of an incident plane wave with the nanophotonic structures. In fact, inside the substrate, due to the complex interplay of local fields produced by the incident wave interacting with the meta-inclusions, we encounter fields that are no longer plane waves (unlike the "simple" chiral substrate case examined in section I). Assuming that the spacing between the meta-inclusions is much shorter than the wavelength, this complex interaction can be simplified by attributing effective constitutive parameters to the nanophotonic substrate. ${ }^{38,39}$ By using these parameters, the substrate is described as a homogeneous matter, which allows for considering plane-wave fields inside the substrate. The second challenge in the analytical treatment is associated with the nearfield interaction of the chiral substrate with the biolayer. We know that the effective parameters are associated with the farfield reflection and transmission of such structures. On the other hand, nanophotonic structures change, both, the intensity and chirality of the light in their vicinity (near field). We define the local intensity- and local chirality-enhancement factors as $\mathcal{F}_{\mathrm{p}}^{\mathrm{loc}}=|\boldsymbol{E}|_{\text {near }}^{2} /|\boldsymbol{E}|_{\text {far }}^{2}$ and $\chi_{\mathrm{p}}^{\mathrm{loc}}=\operatorname{Im}\left\{\boldsymbol{E} \cdot \boldsymbol{H}^{*}\right\}_{\text {near }} / \operatorname{Im}\left\{\boldsymbol{E} \cdot \boldsymbol{H}^{*}\right\}_{\text {far }}$, respectively, where the "near" and "far" subscripts refer to the near- and far-field zones, and " $p$ " can be either $R$ or $L$ denoting the polarization of the input excitation. The near-field (farfield) zone is defined as a volume of the same size as the chiral sample, immediately on top of the substrate (at the end of the physical simulation domain). The chiral sample is placed in these two different zones and the local factors are calculated by using the corresponding field values at each point inside the chiral sample (see SI for more details.). By the so-defined enhancement factors, it can be shown (see SI) that the effective values for the imaginary parts of the permittivity and Pasture parameter of the biolayer are modified by the factors of $\mathcal{F}_{\mathrm{p}}$ and $\chi_{\mathrm{p}}$, which are, respectively, the averaged values of $\mathcal{F}_{\mathrm{p}}^{\text {loc }}$ and $\chi_{\mathrm{p}}^{\text {loc }}$ taken over the chiral sample. It should be noted that, these averaged enhancement factors account for the coupling effects between the nanophotonic substrate and the chiral sample, since the field values $(\boldsymbol{E}$ and $\boldsymbol{H}$ ) used in the calculations of these enhancement factors take into account the presence of both the chiral sample and the substrate. Furthermore, it is to be mentioned that the averaged optical chirality embodies the uniformity of the chiral fields, which is critically important in CD enhancement due to the fact that in realistic implementation of chiral sensing the chiral biolayer is placed uniformly over the nanophotonic structures and not only on the chiral hotspots. ${ }^{36}$

Now we are able to cope with the second challenge in the analytical treatment of the problem at hand, and model the near-field interaction between the nanophotonic substrate and the chiral sample by embedding intensity- and chirality- enhancement factors in the effective permittivity and the effective Pasture parameter of the sensed biolayer. By attributing the obtained effective Pasture parameter and permittivity to the biolayer, and then retrieving the $\mathrm{CD}$ signal from eq 1 , we arrive at the following expression for the total $\mathrm{CD}_{\mathrm{i}}^{\mathrm{m}}$ output,

$$
\begin{aligned}
\mathrm{CD}_{\mathrm{i}}^{\mathrm{m}}= & \mathrm{CD}_{\mathrm{m}}+\left\{\frac{k_{0} w_{\mathrm{i}} \operatorname{Im}\left\{\varepsilon_{\mathrm{i}}\right\}}{2 \sqrt{\operatorname{Re}\left\{\varepsilon_{\mathrm{i}}\right\}}}\left(\mathcal{F}_{\mathrm{L}}-\mathcal{F}_{\mathrm{R}}\right)\right. \\
& \left.+\frac{\mathrm{CD}_{\mathrm{i}}}{2}\left(\chi_{\mathrm{R}}+\chi_{\mathrm{L}}\right)\right\} \operatorname{sech}\left(4 k_{0} w_{\mathrm{m}} \operatorname{Im}\left\{\kappa_{\mathrm{m}}\right\}\right)^{\prime}
\end{aligned}
$$

where $\mathrm{CD}_{\mathrm{m}}$ is the $\mathrm{CD}$ contribution of the nanophotonic substrate (without the biolayer), which can be obtained by inserting its corresponding parameters $\left(\kappa_{\mathrm{m}}, w_{\mathrm{m}}\right)$ in eq 3 . Equation 6 analytically explains two different effects of substrate chiral absorption $\left(k_{0} w_{\mathrm{m}} \operatorname{Im}\left\{\kappa_{\mathrm{m}}\right\}\right)$ in nanophotonic CD spectroscopy. The first effect is that it produces an unwanted background noise of $\mathrm{CD}_{\mathrm{m}}$, which has been considered in previous works, ${ }^{14,32}$ but we herein obtained it analytically, in eq 3. The second effect, which to our knowledge has not been pointed out before, is the attenuation role of the chiral absorption on the output $\mathrm{CD}$, originating from the evanescent nature of the sech function. This is also emphasized in the analysis of section I. Lastly, our closed-form expression in eq 6 elucidates analytically that the total output CD depends not only on the chirality of the biolayer but also on the biolayer's permittivity. This is a restatement of the fact that if the system is illuminated with two fields of different intensities (i.e., as in the case with two different near-field intensity enhancements) the subtraction of their corresponding difference will be nonzero and depends on the permittivity.

Equation 6 and its associated implications are the key findings of the present work, summarizing the contributing factors for improving the $\mathrm{CD}$ signal retrieved from chiral samples using nanophotonic substrates. According to this equation, the optimum nanophotonic-platform candidates for substrate CD spectroscopy are those that feature: $(i)$ small values of $4 k_{0} w_{\mathrm{s}} \operatorname{Im}\left\{\kappa_{\mathrm{m}}\right\}$ to remove $\mathrm{CD}_{\mathrm{m}}$ and make the sech argument zero; (ii) equal intensity-enhancement factors for $\operatorname{RCP}\left(\mathcal{F}_{\mathrm{R}}\right)$ and $\operatorname{LCP}\left(\mathcal{F}_{\mathrm{L}}\right)$ to eliminate the permittivity dependency in eq 6; and (iii) high optical chirality in their near-field zones to maximize the term $\chi_{\mathrm{R}}+\chi_{\mathrm{L}}$. These three requirements help to clarify the trade-offs in the conception of substrate CD-enhancement platforms. The first class of structures can be provided by thin metasurfaces $\left(k_{0} w_{\mathrm{s}} \rightarrow 0\right)$, lossless dielectrics $\left(\operatorname{Im}\left\{\kappa_{\mathrm{m}}\right\}=0\right)$ or achiral $\left(\kappa_{\mathrm{m}}=0\right)$ nanophotonic structures. The second one requires achiral structures, interacting with either RCP or LCP waves in the same manner, thereby leading to $\mathcal{F}_{\mathrm{R}}=\mathcal{F}_{\mathrm{L}}$ in the near-field zone of the structure. The third class can be afforded by, both, chiral or achiral structures, but generally the chiral ones have higher amounts of optical chirality compared to their achiral counterparts. $^{14}$

Based on all of the aforementioned considerations, herein we introduce an achiral dielectric nanophotonic substrate, composed of silicon nanodisks (of refractive index $n_{\mathrm{d}}=3.5$ ), embedded in a homogeneous dielectric with refractive index of $n=1.5$. This proposed platform allows for all of abovementioned features, and has a simplified total CD signal of 
(a)

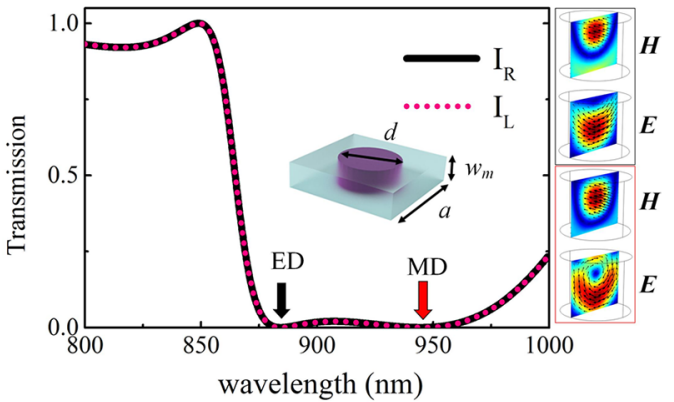

(c)

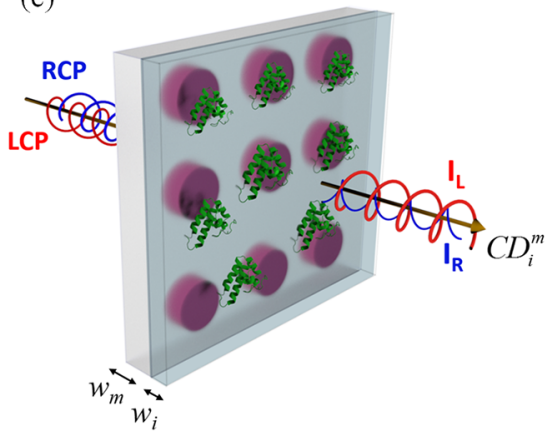

(b)
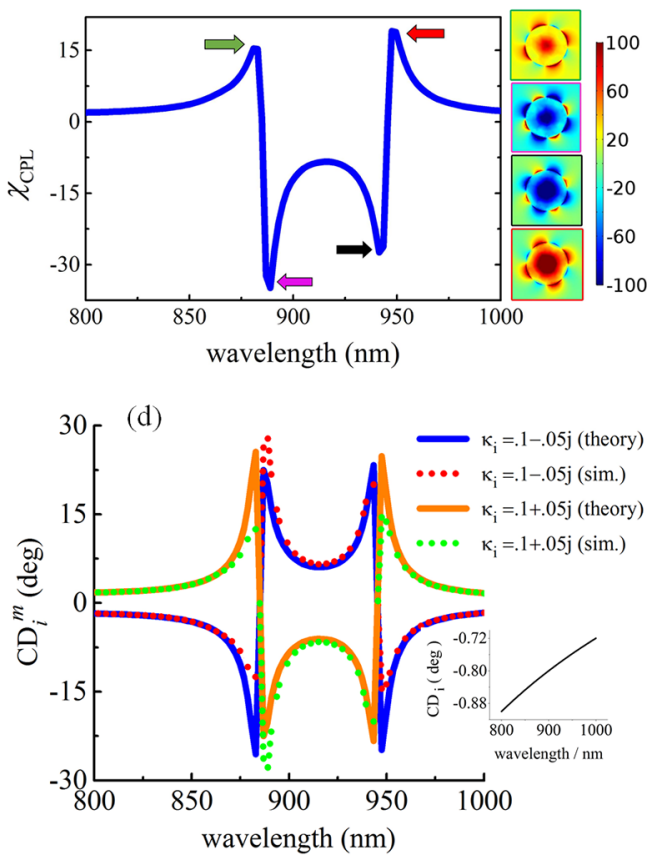

Figure 3. Nanophotonic-substrate CD spectroscopy. (a) Transmission spectra of RCP $\left(I_{\mathrm{R}}\right)$ and LCP ( $\left.I_{\mathrm{L}}\right)$ waves for the proposed achiral dielectric substrate. The structure is composed of silicon nanodisks of diameter $d=290 \mathrm{~nm}$ and height $w_{\mathrm{m}}=220 \mathrm{~nm}$, with a lattice constant of $a=490 \mathrm{~nm}$, and embedded into a homogeneous dielectric of refractive index of $n=1.5$. The inset shows a unit cell of the structure. The transmission spectra show two resonance dips at 885 and $945 \mathrm{~nm}$, which arise from an electric dipole (ED) and a magnetic dipole (MD) response, respectively, of the silicon nanodisks. The mode profiles at ED and MD resonances are shown at the at the central vertical cut of the nanodisk by black and red square panels, respectively. (b) Averaged optical chirality enhancement factor for the proposed nanophotonic substrate. The color panels show the local optical chirality enhancement at the ED and MD resonances, immediately on top of the nanodisk. (c) The chiral sample with refractive index of $n_{\mathrm{i}}=1.45-$ $0.1 j$ and Pasture parameter of $\kappa_{\mathrm{i}}=0.1 \pm 0.05 j$, placed on top of the proposed substrate. The whole system is excited by RCP (blue) and LCP (red) light, and the $C D_{i}^{m}$ at the output side measures the total differential absorbance of the system. (d) Calculated $C D_{i}^{m}$ signal, as obtained by analytic theory and simulations, for two chiral samples with different signs of Pasture parameter. Inset shows the chirality of the biofilm alone.

$$
\mathrm{CD}_{\mathrm{i}}^{\mathrm{m}}=\chi_{\mathrm{CPL}} \mathrm{CD}_{\mathrm{i}}
$$

where $\chi_{\mathrm{CPL}}$ is the chirality-enhancement factor for either RCP or LCP waves. It is evident from eq 7 that this structure features a background-free CD signal, which is only dependent on the handedness of the biolayer, amplified by a factor of $\chi_{\mathrm{CPL}}$.

Figure $3 \mathrm{a}$ presents the transmission spectra for RCP and LCP waves, with the disk diameter $(d)$ and height $\left(w_{\mathrm{m}}\right)$ being 220 and $290 \mathrm{~nm}$, respectively (inset). We note that the two transmissions are identical, implying a vanishing $\mathrm{CD}_{\mathrm{m}}$ background signal. On the other hand, the transmission spectra shows two resonance dips at $\lambda=885$ and $945 \mathrm{~nm}$, which arise from an electric dipole (ED) and magnetic dipole (MD) response of the silicon nanodisks, respectively (cf. field patterns in Figure 3a). Figure 3b shows the chirality-enhancement factor for the proposed platform. It is evident that the ED and MD resonances are well capable of providing high optical chirality at their corresponding wavelengths, as well as providing considerable optical chirality between the two resonances. These chiral near-fields appear in dielectric structures with high refractive index at multipolar Mie resonances. ${ }^{40}$ Thus, this dielectric achiral substrate still offers high optical chirality, which generally characterizes plasmonic chiral structures. It is important to note that at the two sides of both resonances the sign of the optical chirality is reversed, implying a corresponding change to the sign of the CD signal when a biolayer is placed on top of the substrate.
Figure $3 c$ illustrates a chiral sensing configuration by our proposed platform, in which a chiral sample of thickness $20 \mathrm{~nm}$ and Pasture parameter $\kappa_{\mathrm{m}}=0.1-.05 j$ is placed on top of the introduced nanophotonic substrate, and the whole system is excited by RCP (blue) and LCP (red) light. The $\mathrm{CD}_{\mathrm{i}}^{\mathrm{m}}$ at the output side measures the total differential absorbance of the system for RCP and LCP illuminations, which for this special platform arises only from the biolayer chirality $\left(\operatorname{Im}\left\{\kappa_{\mathrm{i}}\right\}\right)$. In Figure $3 \mathrm{~d}$ we have calculated the $\mathrm{CD}_{\mathrm{i}}^{\mathrm{m}}$ signal theoretically (solid blue) and by full-wave simulations (dotted red). It can be seen that the theoretical and the simulation results are in excellent agreement. The inset in Figure $3 \mathrm{~d}$ shows the $\mathrm{CD}$ signal of the biolayer without substrate, which is only about $0.80^{\circ}$. It should be noted that, in addition to removing the background noise and permittivity dependency, this platform provides extremely high $\mathrm{CD}$ enhancement, of the order of 30 at the resonances and 10-20 between the resonances. In order to check that the $\mathrm{CD}$ signal is only dependent on the handedness of the sample, we have studied another biolayer of Pasture parameter $\kappa_{\mathrm{m}}=0.1$ $+0.05 j$. We assumed that the two biolayers are only different in their CD signatures, not in their ORD responses, which corresponds to different signs for the imaginary part of the Pasture parameter. If the platform does not have any background noise and is not permittivity-dependent, then by changing the handedness of the biolayer the $\mathrm{CD}$ signal should be digitally reversed in sign. Figure $3 \mathrm{~d}$ shows the theoretical (solid orange) and simulation (dotted green) results for this second biolayer. It is seen that the simulation and theoretical 
results are again in an excellent agreement, and the CD signal is digitally reversed with respect to the first biolayer, as expected.

In conclusion, the above discussions and results reveal that the herein-developed analytical closed-form expressions of substrate-enhanced chiral sensing accurately characterize the response of these structures, and help to guide their optimal design. Our analysis is inherently capable of treating all kinds of nanophotonic chiral-sensing platforms, including plasmonic or dielectric, chiral or achiral, three-dimensional, or thin surface structures (metasurfaces), and on this basis, we identified an optimal, dielectric, achiral class of such structures for enhanced, background-free chiral sensing. Our results corroborate and extend previously reported aspects of such structures, highlighting overlooked features of substrate-enhanced chiral lightmatter interactions, and providing overall an analytic insight at the trade-offs that are to be accommodated in the optimal design of these intricate configurations.

\section{ASSOCIATED CONTENT}

\section{S Supporting Information}

The Supporting Information is available free of charge on the ACS Publications website at DOI: 10.1021/acsphotonics.8b00270.

S1: CD Signal of a chiral bio-layer. S2: CD signal of a bio-layer placed on top of a simple chiral substrate. S3: Effective values of permittivity and Pasture parameter for a chiral sample placed on top of a nanophotonic substrate. Appendix S1: Fields in isotropic chiral media. Appendix S2: Poynting's theorem for chiral media (PDF).

\section{AUTHOR INFORMATION}

\section{Corresponding Author}

*E-mail: hatice.altug@epfl.ch.

\section{ORCID 1}

E. Mohammadi: 0000-0001-6029-2672

\section{Notes}

The authors declare no competing financial interest.

\section{ACKNOWLEDGMENTS}

We acknowledge the support of European Union Horizon 2020 Framework Programme for Research and Innovation under grant agreements no. FETOPEN-737071 (ULTRACHIRAL project), no. 777714 (NOCTURNO project) and EPFL Fellows" Co-found Marie-Curie Fellowship for K.L.T. Also, funding from Ministry of Science, Research and Technology of Islamic Republic of Iran is gratefully acknowledged.

\section{REFERENCES}

(1) Barron, L. D. Chirality and life. Space Sci. Rev. 2008, 135 (1-4), $187-201$.

(2) Dobson, C. M. Protein folding and misfolding. Nature 2003, 426 (6968), 884-890.

(3) Davies, N. M.; Xiao Wei, T. Importance of chirality in drug therapy and pharmacy practice: Implications for psychiatry. Advances in Pharmacy 2003, 3, 242-252.

(4) Smith, S. W. Chiral toxicology: it's the same thing-only different. Toxicol. Sci. 2009, 110, 4-30.

(5) Boriskina, S., Nikolay, I. Z., Eds. Singular and Chiral Nanoplasmonics; CRC Press, 2014.

(6) Barron, L. D. Molecular Light Scattering and Optical Activity; Cambridge University Press, 2004.
(7) Nordén, B.. Circular Dichroism and Linear Dichroism; Oxford University Press, 1997; Vol. 1.

(8) Lindell, I. V.; Sihvola, A. H.; Tretyakov, S. A.; Viitanen, A. J. Electromagnetic Waves in Chiral and Bi-Isotropic Media; Artech Print on Demand, 1994.

(9) Tsakmakidis, K. L.; Boyd, R. W.; Yablonovitch, E.; Zhang, X. Large spontaneous-emission enhancements in metallic nanostructures: towards LEDs faster than lasers. Opt. Express 2016, 24 (16), 1791627.

(10) Tsakmakidis, K. L.; Hess, O.; Boyd, R. W.; Zhang, X. Ultraslow waves on the nanoscale. Science 2017, 358 (6361), eaan5196.

(11) Valev, V. K.; Baumberg, J. J.; Sibilia, C.; Verbiest, T. Chirality and chiroptical effects in plasmonic nanostructures: fundamentals, recent progress, and outlook. Adv. Mater. 2013, 25 (18), 2517-34.

(12) Hendry, E.; Mikhaylovskiy, R. V.; Barron, L. D.; Kadodwala, M.; Davis, T. J. Chiral electromagnetic fields generated by arrays of nanoslits. Nano Lett. 2012, 12 (7), 3640-4.

(13) Luo, Y.; Chi, C.; Jiang, M.; Li, R.; Zu, S.; Li, Y.; Zheyu, F. Plasmonic Chiral Nanostructures: Chiroptical Effects and Applications. Adv. Opt. Mater. 2017, 5, 170040.

(14) Schäferling, M. Chiral Nanophotonics: Chiral Optical Properties of Plasmonic Systems; Springer, 2016; Vol. 205.

(15) Gansel, J. K.; Thiel, M.; Rill, M. S.; Decker; Bade, K.; Saile, V.; von Freymann, G.; Linden, S.; Wegener, M. Gold helix photonic metamaterial as broadband circular polarizer. Science 2009, 325 (5947), 1513-1515.

(16) Esposito, M.; Tasco, V.; Cuscunà, M.; Todisco, F.; Benedetti, A.; Tarantini, I.; De Giorgi, M.; Sanvitto, D.; Passaseo, A. Nanoscale 3D chiral plasmonic helices with circular dichroism at visible frequencies. ACS Photonics 2015, 2, 105-114.

(17) Bettina, F.; Yin, X.; Schäferling, M.; Zhao, J.; Hein, S. M.; Braun, P. V.; Giessen, H. Large-area 3D chiral plasmonic structures. ACS Nano 2013, 7 (7), 6321-6329.

(18) Decker, M.; Ruther, M.; Kriegler, C. E.; Zhou, J.; Soukoulis, C. M.; Linden, S.; Wegener, M. Strong optical activity from twisted-cross photonic metamaterials. Opt. Lett. 2009, 34, 2501-2503.

(19) Zhao, Y.; Belkin, M. A.; Alù, A. Twisted optical metamaterials for planarized ultrathin broadband circular polarizers. Nat. Commun. 2012, 3, 870 .

(20) Hentschel, M.; Martin, S.; Thomas, W.; Na, L.; Harald, G. Three-dimensional chiral plasmonic oligomers. Nano Lett. 2012, 12, 2542-2547.

(21) Guerrero-Martínez, A.; Auguié, B.; Alonso-Gómez, J. L.; Džolić, Z.; Gómez-Graña, S.; Žinić, M.; Cid, M. M.; Liz-Marzán, L. M. Intense Optical Activity from Three-Dimensional Chiral Ordering of Plasmonic Nanoantennas. Angew. Chem., Int. Ed. 2011, 50, 54995503.

(22) Fan, Z.; Alexander, O. G. Plasmonic circular dichroism of chiral metal nanoparticle assemblies. Nano Lett. 2010, 10, 2580-2587.

(23) Hendry, E.; Carpy, T.; Johnston, J.; Popland, M.; Mikhaylovskiy, R. V.; Lapthorn, A. J.; Kelly, S. M.; Barron, L. D.; Gadegaard, N.; Kadodwala, M. Ultrasensitive detection and characterization of biomolecules using superchiral fields. Nat. Nanotechnol. 2010, 5, 783-787.

(24) Kwon, D.-H.; Pingjuan, L. W.; Werner, D. H. Optical planar chiral metamaterial designs for strong circular dichroism and polarization rotation. Opt. Express 2008, 16, 11802-11807.

(25) Kaschke, J.; Martin, W. Gold triple-helix mid-infrared metamaterial by STED-inspired laser lithography. Opt. Lett. 2015, 40, 3986-3989.

(26) Yoo, S.; Cho, M.; Q-Han, P. Globally enhanced chiral field generation by negative-index metamaterials. Phys. Rev. B: Condens. Matter Mater. Phys. 2014, 89 (16), 161405.

(27) Davis, T. J.; Hendry, E. Superchiral electromagnetic fields created by surface plasmons in nonchiral metallic nanostructures. Phys. Rev. B: Condens. Matter Mater. Phys. 2013, 87 (8), 085405.

(28) García-Etxarri, A.; Dionne, J. A. Surface-enhanced circular dichroism spectroscopy mediated by nonchiral nanoantennas. Phys. Rev. B: Condens. Matter Mater. Phys. 2013, 87 (23), 235409. 
(29) Tang, Y.; Cohen, A. E. Enhanced enantioselectivity in excitation of chiral molecules by superchiral light. Science 2011, 332 (6027), 333-336.

(30) Tang, Y.; Cohen, A. E. Optical chirality and its interaction with matter. Phys. Rev. Lett. 2010, 104, 163901.

(31) Schäferling, M.; Dregely, D.; Hentschel, M.; Giessen, H. Tailoring enhanced optical chirality: design principles for chiral plasmonic nanostructures. Phys. Rev. X 2012, 2 (3), 031010.

(32) Zhao, Y.; Askarpour, A. N.; Sun, L.; Shi, J.; Li, X.; Alù, A. Chirality detection of enantiomers using twisted optical metamaterials. Nat. Commun. 2017, 8, 14180.

(33) Yin, X.; Schäferling, M.; Metzger, B.; Giessen, H. Interpreting chiral nanophotonic spectra: the plasmonic Born-Kuhn model. Nano Lett. 2013, 13 (12), 6238-43.

(34) Govorov, A. O.; Fan, Z.; Hernandez, P.; Slocik, J. M.; Naik, R. R. Theory of circular dichroism of nanomaterials comprising chiral molecules and nanocrystals: plasmon enhancement, dipole interactions, and dielectric effects. Nano Lett. 2010, 10 (4), 1374-82.

(35) Schäferling, M.; Engheta, N.; Giessen, H.; Weiss, T. Reducing the complexity: Enantioselective chiral near-fields by diagonal slit and mirror configuration. ACS Photonics 2016, 3 (6), 1076-84.

(36) Nesterov, M. L.; Yin, X.; Schäferling, M.; Giessen, H.; Weiss, T. The role of plasmon-generated near fields for enhanced circular dichroism spectroscopy. ACS Photonics 2016, 3, 578.

(37) Poulikakos, L. V.; Gutsche, P.; McPeak, K. M.; Burger, S.; Niegemann, J.; Hafner, C.; Norris, D. J. Optical chirality flux as a useful far-field probe of chiral near fields. ACS Photonics 2016, 3 (9), 161925.

(38) Zhao, R.; Koschny, T.; Soukoulis, C. S. Chiral metamaterials: retrieval of the effective parameters with and without substrate. Opt. Express 2010, 18, 14553-14567.

(39) Chen, X.; Grzegorczyk, T. M.; Wu, B.-I.; Pacheco, J.; Kong, J. A. Robust method to retrieve the constitutive effective parameters of metamaterials. Physical Review E 2004, 70, 016608.

(40) Ho, C. S.; Garcia-Etxarri, A.; Zhao, Y.; Dionne, J. Enhancing enantioselective absorption using dielectric nanospheres. ACS Photonics 2017, 4 (2), 197-203. 